\title{
A Model for Measuring Ambulatory Access to Care Recovery after Disasters
}

\author{
Tiffany A. Radcliff, PhD, Karen Chu, MS, Claudia Der-Martirosian, PhD, and \\ Aram Dobalian, $\mathrm{PhD}$, JD, $\mathrm{MPH}$
}

Introduction: Although little research has examined impacts of disasters on scheduled ambulatory care services, routine care delivery is important for emergency planning and response because missed or delayed care can lead to more urgent care needs. This article presents potential measures of ambulatory care recovery and resilience and applies the measures to data around a recent disaster.

Methods: We conceptualize "ambulatory care recovery" as the change in median business days to complete appointments that were canceled, and "ambulatory care resiliency" as the change in percentage of completed appointments in time frames before, during, and after disasters. Appointments data from Veterans Affairs (VA) clinics were examined around a category 4 hurricane that affected a coastal area with a substantial veteran population.

Results: For the disaster studied, ambulatory care resilience was associated with geographic proximity to the storm's impact. Primary care recovery was longer in locations closest to storm landfall. This research indicates the usefulness of routine appointments data in emergency planning.

Conclusion: Quantifying care disruptions around disasters is an important step in assessing interventions to improve emergency preparedness and response for clinics. The illustrative example of measures captured the disaster event duration and severity in relation to ambulatory care appointments. (J Am Board Fam Med 2018;31:252-259.)

Keywords: Ambulatory Care, Disasters, Emergency Preparedness, Mental Health, Primary Health Care, Telemedicine

Recent unprecedented disasters have renewed concerns initially raised after Hurricane Katrina (in 2005) about disaster preparedness, response, and recovery for communities, individuals, and health care systems. Images of devastated neighborhoods, prolonged electricity outages, toxic chemical spills, and medical evacuations provide vivid depictions of health hazards that increase the immediate and long-term medical needs for populations affected by a disaster. Disasters have been linked to increased injuries, mental health concerns, chemical exposures, and cardiovascular stressors-all of

This article was externally peer reviewed.

Submitted 2 June 2017; revised 10 November 2017; accepted 13 November 2017.

From the Veterans Emergency Management Evaluation Center (VEMEC), US Department of Veterans Affairs, North Hills, CA (TAR, KC, CD-M, AD); the Department of Health Policy and Management, School of Public Health, Texas A\&M University, College Station, TX (TAR); and the Division of Health Systems Management and Policy, School of Public Health, University of Memphis, Memphis, TN (AD). which can lead to a surge in demand for health care services; disasters may also create temporary disruptions in routine service delivery, resulting in barriers to completing previously scheduled appointments. ${ }^{1-3}$ Scheduled ambulatory care remains the most-used type of care in the United States. Because disasters may jeopardize care continuity if patients evacuate or need to seek care outside of their planned encounter, disaster-related care disruptions may result in exacerbated chronic conditions or limit preventive care and lead to more

Funding: This material is based upon work supported by the Department of Veterans Affairs, Veterans Health Administration, Office of Patient Care Services.

Conflict of interest: none declared.

Disclaimer: The views expressed in this article are those of the authors and do not necessarily reflect the position or policy of the Department of Veterans Affairs or the US government.

Corresponding author: Tiffany A. Radcliff, PhD, Department of Health Policy and Management, School of Public Health, Texas A\&M University, 212 Adriance Lab Road, College Station, TX 77843-1266 (E-mail: radcliff@tamhsc.edu). 
expensive emergency department or hospital-based care. $^{4-9}$ Measures of ambulatory clinic recovery and resilience have not been standardized, but they are increasingly important as natural and humanmade emergencies become more frequent and severe.

During disasters, most missed ambulatory care appointments are beyond the immediate control of the patient or provider. The uncertainty about when and where disasters occur creates a need for advance preparation by clinics to ensure continuity of operations and efficient recovery. Most systems of care have the capability to notify patients of clinic closures or cancellations, and many list alternative resources for more urgent care needs. In addition, providers and systems have tools needed to identify patterns of missed appointments. Putting these types of information together can provide practical insight to minimize future disasterrelated disruptions. This article presents measures of ambulatory care recovery and resilience that rely on routine appointment records and discusses implications for future disaster planning and research. In doing so, this article supports information needed to understand the role of disasters in perpetuating disparities for vulnerable populations and patients with chronic care needs. ${ }^{10-12}$ To illustrate the proposed measures in practice, we analyzed appointment system data from US Department of Veterans Affairs (VA) clinics to compare the measures for areas affected and not affected by a significant natural disaster.

\section{Methods}

The study team developed measures and methods to assess recovery and resilience according to missed and completed appointments in ambulatory care. A retrospective cohort observational study design that included clinics in affected areas and a comparison group of unaffected clinics was then used to determine the feasibility of these measures for routine health care operations and emergency planning. The measures were compared for time frames before, during, and after a disaster. Approval to analyze administrative data for this study was received from the VA Greater Los Angeles Healthcare System Institutional Review Board.

\section{Measures}

The measure of ambulatory care recovery was developed to capture restoration of services around care disruptions, whereas the resiliency measure was designed to capture deviations from prescribed or identified "norms" in routine care operations that are comparable across clinics and over time.

\section{Ambulatory Care Recovery}

"Recovery" for ambulatory clinics refers to the time required for specific patients to complete appointments canceled by the clinic. For example, if a clinic cancels a patient's wellness examination, the number of business days until that patient completes a scheduled wellness examination measures the recovery of that appointment. The mean or median number of days to complete clinic cancellations is then calculated by time frame (week, month, before/after the disaster). Mean values reflect appointments that have extended time frames for completion (outliers), which may include factors beyond the capacity of the clinic to reschedule, whereas median values are the 50th percentile (middle) of the range for recovered appointments. Equations (1) and (2) provide the general calculation details for the recovery metric.

Days to complete appointment

$$
=\text { Date completed }- \text { Date missed }
$$

Recovery $=$ Mean (or median) business days to complete missed appointments in time period $(t)$

To gauge recovery, results from Eq. (2) are compared across time periods as follows:

$$
\begin{aligned}
& \text { Change in Recovery }{ }_{t, t-1} \\
& \qquad=\text { Recovery }_{t}-\text { Recovery }_{t-1}
\end{aligned}
$$

where $t$ is the reference time period and $t-1$ is either the previous time frame (eg, the week before the disaster occurred) or a general reference time period (eg, the same week as $t$ the year before). A positive difference in the mean or median number of business days needed to complete appointments indicates longer delays in time period $t$ compared with time period $t-1$. If desired, recovery can be expressed as a percentage change: 
Change in recovery $y_{t, t-1}(\%)$

$$
=\frac{\text { Recovery }_{t}-\text { Recovery }_{t-1}}{\text { Recovery }_{t-1}}
$$

\section{Ambulatory Care Resiliency}

While recovery measures focus on each missed appointment, resiliency is related to overall proportions of completed care. The percentage of completed appointments during a given time frame is provided in Eq. (4):

Completed appointments ${ }_{t}(\%)$

$$
=\frac{\text { Schedule appointments completed } t(n)}{\text { Appointments scheduled }_{t}(N)}
$$

where $t$ indicates the reference time period (eg, the week in which the disaster occured). The measure of resiliency is calculated as the change in the percentage of completed appointments across 2 time frames:

$$
\begin{array}{r}
\text { Resiliency }_{t}=\text { Completed Appointments }_{t}(\%) \\
- \text { Completed Appointments }_{t-1}(\%)
\end{array}
$$

In other words, highly resilient clinics would achieve at least the same percentage of completed appointments as in the time frame before the disaster.

\section{Ambulatory Care Recovery and Resiliency Metrics: Illustrative Example}

To illustrate these metrics in practice, we used existing VA administrative records around a category 4 hurricane that struck the Gulf Coast region of the United States. VA appointment records included clinic locations directly affected by the storm and other locations not affected by the storm.

\section{Study Sample}

We studied 9 VA "stations" with 10 VA medical centers (VAMCs) and 36 community clinics that offered ambulatory care services during the disaster time frame. From among all appointments scheduled at these clinic locations we selected appointments for the 25 weeks ranging from 12 weeks before to 12 weeks after hurricane landfall and limited these appointments to a subsample of patients with a recent history (within 12 months of storm landfall) of accessing the VA for routine care services or prescribed medications. We included appointments corresponding to the most frequent ambulatory care clinic codes (excluding diagnostic services) plus telehealth, as it offers an alternative mode for completing appointments. Appointments were grouped into the following 5 categories:

1. Primary care: 1 clinic code (primary care clinic)

2. Mental health: 5 clinic codes (mental health clinic, posttraumatic stress disorder group, posttraumatic stress disorder individual, substance abuse group, substance abuse individual)

3. Specialty care: 5 clinic codes (cardiology, ophthalmology, urology, orthopedics, dermatology)

4. Other health professions: 6 clinic codes (dental, optometry, physical therapy, audiology, podiatry, clinical pharmacy)

5. Telehealth: 32 clinic codes (telephone-based triage, diagnosis, or treatment)

Disposition of each appointment was recorded by the clinic. The following disposition categories were used to measure recovery and resilience: completed as planned, canceled by the clinic, no longer needed, or missed (either canceled or no-show) by the patient. Appointments that were no longer needed or had missing disposition information were excluded from further study.

Affected verses not affected clinic designation was determined by location in geographic areas where storm damage occurred or where there existed shared administrative structures, services, and leadership with an affected VAMC or station. Based on this definition, all clinic locations in a single VA station where the storm made landfall were classified as affected by this disaster. The affected locations included 1 large VAMC in a large metropolitan area and 4 VA outpatient clinics; the locations ranged from the coast to approximately 100 miles inland. All other clinics in the same Veterans Integrated Service Network, which did not have direct geographic or administrative overlap with the storm's landfall, were classified as not affected and served as the comparison group of clinics.

Recovery measures were calculated as the change in median days to completed appointments 
Table 1. Sample Sizes and Distribution of Veterans Affairs (VA) Appointments around Disaster Impact (Week 0)

\begin{tabular}{lcrr}
\hline & Unaffected Areas & Affected Areas & Total (n) \\
\hline Unique patients (weeks -12 to 12) & $197,724(78.0)$ & $56,790(22.0)$ & 254,514 \\
Appointments & & & $302,490(25.2)$ \\
$\quad$ Total (weeks -12 to 12) & $897,405(74.8)$ & $122,205(25.4)$ & $1,199,895$ \\
Before the disaster (weeks -12 to -3$)$ & $358,479(74.6)$ & $48,926(25.4)$ & 480,684 \\
During the disaster (weeks -2 to 1) & $143,450(74.6)$ & $131,359(24.9)$ & 192,376 \\
After the disaster (weeks 2-12) & $395,476(75.1)$ & & 526,835 \\
Appointment type & & $128,535(42.5)$ & \\
Primary care & $361,222(40.3)$ & $65,966(21.8)$ & 389,757 \\
Mental health & $178,782(19.9)$ & $38,579(12.8)$ & 244,748 \\
Non-MD providers & $192,708(21.5)$ & $56,295(18.6)$ & 231,287 \\
Specialty care & $137,853(15.4)$ & $13,115(4.3)$ & 194,148 \\
Telehealth & $26,840(3.0)$ & 39,955 \\
\hline
\end{tabular}

Data are no. (\%) unless otherwise indicated. For this analysis, affected areas included 1 VA medical center plus 4 distinct outpatient clinic locations, whereas unaffected areas included 9 VA medical centers and 32 distinct outpatient clinic locations.

for the 5 categories of clinic codes listed above. Appointment system records were used to calculate resiliency as the percentage of completed appointments by week and the change in the percentage of completed appointments by week for VA clinics. Descriptive results for recovery and resiliency are presented along with a measure of the importance of differences relative to the overall facility operations. For recovery, a 5-business day difference in median days to appointment completion was selected as the benchmark because it represents 1 week of clinic appointments. In assessing resiliency, we consulted with VA clinic leaders to determine what change in the percentage of appointments would be important from a managerial perspective. Using this approach for the illustrative example, we specified that a 5-percentage point reduction in completed appointments per week compared with the completion rates before the storm was operationally significant in VA ambulatory care, whereas a $10-$ percentage point reduction was substantial.

\section{Results}

We identified approximately 1.2 million appointment records for 254,514 unique patients during the 25week time frame, with nearly $25 \%$ of the appointments corresponding to the affected VAMC and clinics and $75 \%$ to the VAMCs and clinics in geographic areas not affected by the disaster (Table 1). Across all clinic categories in our sample, $41 \%$ of the appointments were for primary care, $20 \%$ were for mental health, $19 \%$ for other health professions, $16 \%$ for specialty care, and $3 \%$ for telehealth ser- vices. Table 2 provides descriptive characteristics of the patients in the study sample.

Table 3 presents the median days to recovery for the VAMC location compared with a time frame before the storm. In the time frame before the storm (weeks -12 to -3 ), the median time to completion for a primary care visit canceled by the clinic was 45 days (9 business weeks). The shorter times to completion in the weeks around and after the storm represent improvements relative to the time frame before the storm. Completion times increased from 23 to 30 days for mental health (before vs during the storm) but were within a 1-week difference for the weeks after landfall. Results for non-MD providers, specialty care, and telehealth indicated either slight decreases or temporary increases in recovery times.

Table 4 provides the resiliency metrics in relation to the $5 \%$ and $10 \%$ benchmarks. For clinics located in areas not affected by the storm, appointment completion rates remained largely consistent across the entire study time frame, resulting in resiliency measures that do not vary around the storm. Several clinics in affected areas experienced $>10 \%$ changes in appointment completion rates during the study time frame, with clinics closest to the coast (ie, clinics B and D) showing the largest declines around the storm, followed by a rapid increase in the percentage of completed appointments the following week and minimal variation in weekly percentages of completed appointment by week 5. The percentage of completed appointments is lower for clinics in the affected areas ver- 
Table 2. Demographic Characteristics among Those with Scheduled Veterans Affairs Appointments around a Natural Disaster (Weeks -12 to 12, with Storm Landfall during Week 0)

\begin{tabular}{|c|c|c|c|}
\hline & $\begin{array}{l}\text { Unaffected Areas } \\
(\mathrm{n}=197,724)\end{array}$ & $\begin{array}{l}\text { Affected Areas } \\
(\mathrm{n}=56,790)\end{array}$ & $\begin{array}{l}\text { Statistically } \\
\text { Significant }^{*}\end{array}$ \\
\hline Age (years), week 0 & & & Yes \\
\hline$<45$ & 8.8 & 10.9 & \\
\hline $45-64$ & 46.1 & 48.5 & \\
\hline$\geq 65$ & 45.1 & 40.6 & \\
\hline Sex & & & Yes \\
\hline Male & 94.5 & 93.0 & \\
\hline Female & 4.7 & 6.1 & \\
\hline Missing/unclear & 0.8 & 0.8 & \\
\hline Race/ethnicity & & & Yes \\
\hline White/non-Hispanic & 66.1 & 54.2 & \\
\hline Hispanic & 0.2 & 1.5 & \\
\hline African American & 12.6 & 19.7 & \\
\hline Other & 1.7 & 0.9 & \\
\hline Missing & 19.4 & 23.7 & \\
\hline Service connection & & & Yes \\
\hline$<50 \%$ & 72.5 & 76.1 & \\
\hline$>50 \%$ & 27.5 & 23.8 & \\
\hline Dual Medicaid & 0.1 & 0.1 & No \\
\hline Dual Medicare & 49.5 & 42.4 & Yes \\
\hline Missed appointment in past 12 months & 33.9 & 43.8 & Yes \\
\hline Veterans Affairs inpatient stay in past 12 months & 9.7 & 11.2 & Yes \\
\hline
\end{tabular}

Data are percentages. For this analysis, affected areas included 1 Veterans Affairs medical center (VAMC) plus 4 distinct outpatient clinic locations, whereas unaffected areas included 9 VAMCs and 32 distinct outpatient clinic locations.

${ }^{*} P<.001, \chi^{2}$ or 2 -sided $t$ test.

sus the unaffected locations for the weeks leading up to the storm (week 0), whereas the reduction in the percentage of completed appointments from the week before the storm through the week after the storm for these areas is dramatic but rapidly increases in the weeks after landfall. For unaffected areas, the percentage of completed appointments remained largely consistent throughout the study time frame, with approximately $70 \%$ of appointments completed each week. For clinics in both affected and unaffected locations, the numbers and percentages of appointments that were no-shows or canceled by the patient were stable over time and consistently represented the majority of the appointments missed. The exception to this was that appointments canceled by the clinic represented the majority of missed appointments for clinics in affected areas for the weeks immediately around landfall (week 0).

\section{Discussion}

A recent call was made for disaster research to be "made more precise" by using transdisciplinary en- deavors to mitigate the health consequences of atrisk populations. ${ }^{13}$ A related concern is that baseline data for determining consequences of disasters are missing. This article presents a simple, flexible, and feasible approach to quantify ambulatory care recovery and resiliency before, during, and after disasters and presents an illustration of the metrics applied to a major disaster for 1 health care system that provides primary care to vulnerable patients with significant health care needs and with historic barriers to accessing care in other health systems. While conceptually and mathematically straightforward, these types of calculations support information needs for providers and population health that are increasingly important as disasters become both more frequent and more severe.

The illustrative example suggests that the storm studied had an impact that was swift and severe for a subset of clinics, with resiliency metrics indicating a differential effect for clinics closest to the coast. Most clinics in affected areas achieved appointment completion percentages that matched or exceeded 

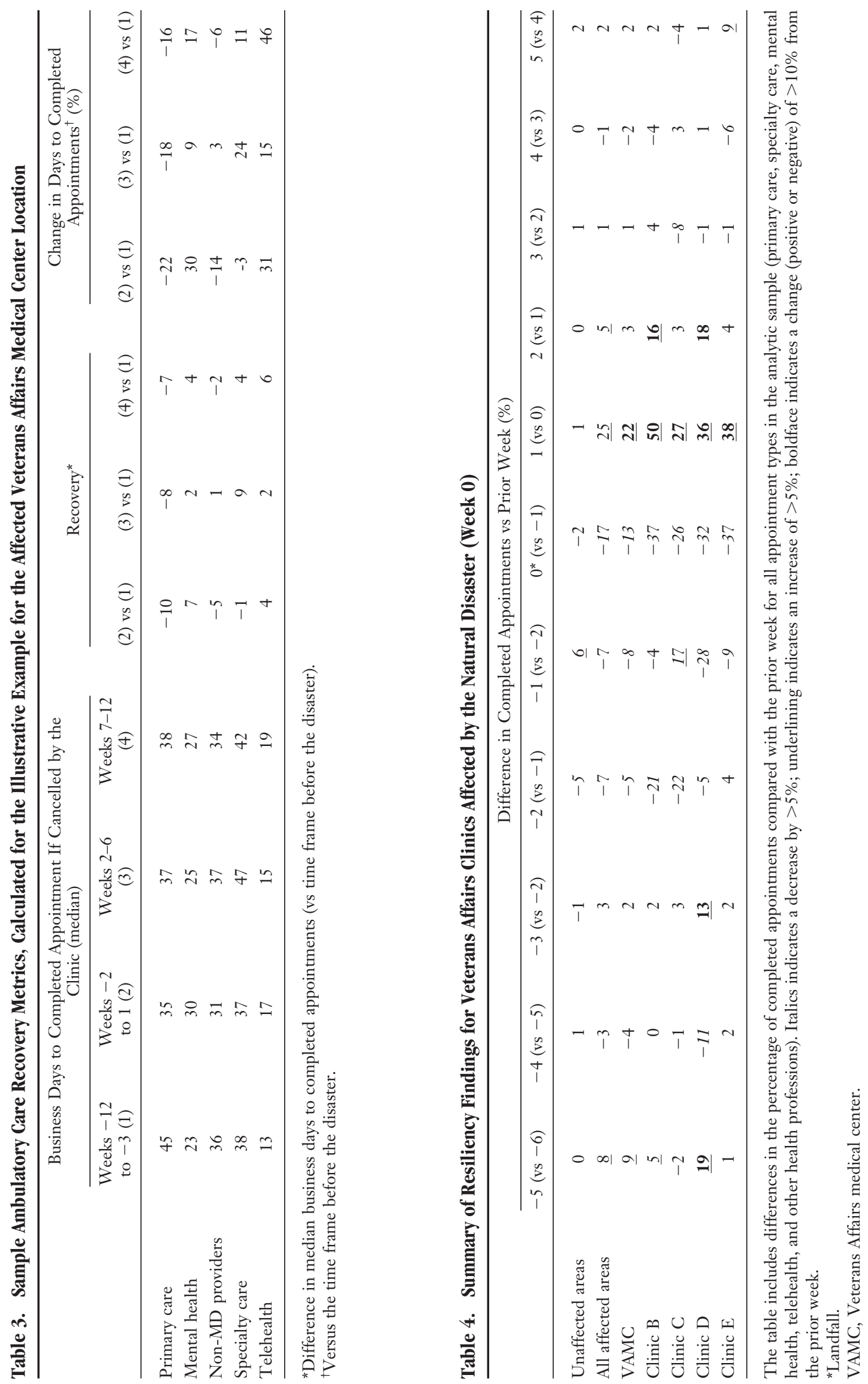
prestorm levels within 2 weeks of the storm. By contrast, the appointment completion percentages for clinics in locations not affected by the storm remained stable over time, suggesting the resiliency metric captures both the event and the relative severity of the event.

The programming of the proposed recovery and resiliency metrics presented in this article can be implemented as routine measures. When services are disrupted during major disasters, these measures can be automatically calculated to provide real-time data to key stakeholders. For example, clinics could use baseline data and data from previous disaster events to identify particular patients or patient subgroups particularly at risk for poor care continuity and proactively recover those appointments. While useful in family medicine clinics and other ambulatory settings, the measures presented in this article are inherently flexible. For example, the metrics could be tailored to represent completion of canceled appointments for each unit within a health system. Alternatively, a composite measure of overall recovery and resilience for entire health care systems could be used to identify a meaningful threshold for implementing interventions to provide alternative care locations for care that was disrupted by a disaster or to prioritize rescheduling for patients at greater risk for negative consequences related to missed ambulatory visits.

Preliminary results of these metrics were presented to a variety of clinicians and managers, who consistently noted that both scheduling and rescheduling patients is not a simple task. Complications for the process can include accommodating a patient's desire to see the same clinician (eg, for continuity of care and to maintain involvement with a "medical home") versus the first available slot with any clinician. This suggests that delays of $\geq 30$ business days to complete a missed appointment may reflect the next appointment available with the desired clinician that is also convenient for the patient, rather than the unavailability of any appointment slot.

Future work should examine whether recent trends in practice affiliation systems and the "franchising" of primary care clinics has reduced disaster-related care disruptions; more family practice providers may have linked medical records, shared resources, and integrated scheduling systems that are similar to the integration found in VA records. The Medical Group Management Association con- ducted a poll in August 2017 (the same month as Hurricanes Harvey and Irma) that suggested that $78 \%$ of practices had an emergency preparedness plan in place. ${ }^{14}$ While the majority of practices reported having a plan, clinics may not have time or resources available when disasters occur to actively prioritize rescheduling missed appointments. This study offers practical metrics to measure near realtime ambulatory care recovery and resiliency around emergency events using appointment disposition data to calculate measures for comparison across clinics and locations, and over time.

This study has limitations. The studied hurricane was a single event and disrupted care for a short period of time. The utility of these metrics should be examined during different crises with longer and shorter durations. Future research could examine differences in ambulatory care resilience and recovery for events that have disaster warnings (eg, hurricanes), where we would expect to see some anticipatory changes in appointments, compared with those that do not (eg, earthquakes), where routine care before the event would follow typical patterns. The recovery and resiliency metrics presented in our example used the time frame before the disaster as the comparator for assessing potential deficits in completing scheduled care. Other organizations may have a different standardized benchmark for setting their time frame(s). Also, our example focused only on VA clinics. VA patients often do not have health insurance and may be limited in their financial ability to receive care from outside the VA. As such, other health systems may experience somewhat different recovery and resiliency metrics depending on whether postdisaster care is affected across a larger region or only selectively at certain facilities within a region. The measures presented in this article were developed for use around disasters and have not yet been tested for other operations and planning purposes. We relied on prestorm values as our comparison, and we recognize that this metric may capture factors other than disasters that delay completing care. Nevertheless, the measure of resiliency, when applied to the VA's appointment data, demonstrated good properties in capturing the disaster, event duration, and event severity.

Quantifying care disruption is an important step in being able to assess the effectiveness of interventions after future disasters, for example, deploying labor from outside the community to temporarily 
supplement routine care needs that were disrupted by the storm. Accordingly, the use of standardized metrics such as this could improve recovery and resiliency for providers and the communities they serve.

To see this article online, please go to: http://jabfm.org/content/ 31/2/252.full.

\section{References}

1. Zarifeh J, Mulder R. Natural disasters and the risk of cardiovascular disease. In: Alvarenga ME, Byrne D, eds. Handbook of psychocardiology. Singapore: Springer Singapore; 2016: 283-302.

2. Hick JL, Hanfling D, Burstein JL, et al. Health care facility and community strategies for patient care surge capacity. Ann Emerg Med 2004;44:253-61.

3. Krol DM, Redlener M, Shapiro A, Wajnberg A. A mobile medical care approach targeting underserved populations in post-Hurricane Katrina Mississippi. J Health Care Poor Underserved 2007;18:331-40.

4. Shapiro MF. No-show patients and the triple aim. J Gen Intern Med 2015;30:1392-3.

5. Mehrotra A. The convenience revolution for treatment of low-acuity conditions. JAMA 2013;310: 35-6.

6. Bech M. The economics of non-attendance and the expected effect of charging a fine on non-attendees. Health Policy 2005;74:181-91.

7. Boos E, Bittner M, Kramer M. A profile of patients who fail to keep appointments in a Veterans
Affairs primary care clinic. Wis Med J 2016;115: 185-90.

8. Partin MR, Gravely A, Gellad ZF, et al. Factors associated with missed and cancelled colonoscopy appointments at Veterans Health Administration facilities. Clin Gastroenterol Hepatol 2016;14:259-67.

9. LaGanga LR, Lawrence SR. Clinic overbooking to improve patient access and increase provider productivity. Decis Sci 2007;38:251-76.

10. Runkle JD, Brock-Martin A, Karmaus W, Svendsen ER. Secondary surge capacity: a framework for understanding long-term access to primary care for medically vulnerable populations in disaster recovery. Am J Public Health 2012;102:e24-32.

11. Tsao JCI, Dobalian A, Wiens BA, Gylys JA, Clawson A, Brooks R. PTSD and substance use: unrecognized sequelae of bioterrorism in primary care providers. South Med J 2006;99:817-22.

12. Dobalian A, Tsao JCI, Putzer GJ, Menendez SM. Improving rural community preparedness for the chronic health consequences of bioterrorism and other public health emergencies. J Public Health Manag Pract 2007;13:476-80.

13. Shultz JM, Galea S. Preparing for the next Harvey, Irma, or Maria - addressing research gaps. N Engl J Med 2017;377:1804-6.

14. MGMA. Many health leaders report they have an emergency preparedness plan. MGMA Stat. August 29, 2017. Available from: https:/www.mgma.com/ industry-data/polling/mgma-stat-archives/do-youhave-an-emergency-preparedness-plan. Accessed November 10, 2017. 